\title{
Transport Theory for a Two-Flavor Color Superconductor
}

\author{
Daniel F. Litim and Cristina Manuel \\ Theory Division, CERN, CH-1211 Geneva 23, Switzerland
}

(Received 14 March 2001; published 12 July 2001)

\begin{abstract}
QCD with two light-quark flavors at high baryonic density is a color superconductor. The diquark condensate breaks the SU(3) gauge symmetry down to an SU(2) subgroup. We study thermal fluctuations of the superconductor for temperatures below the gap. These are described by a simple transport equation. In the collisionless limit and close to equilibrium, it gives rise to the "hard superconducting loop" effective theory for the SU(2) gauge fields. This theory describes Debye screening and Landau damping of the gauge fields in the presence of the diquark condensate. We explain how our effective theory follows to one-loop order from quantum field theory. Our approach provides a convenient starting point for the computation of transport coefficients of the two-flavor color superconductor.
\end{abstract}

DOI: 10.1103/PhysRevLett.87.052002

Quantum chromodynamics (QCD) under extreme conditions displays a very rich phase structure. At low temperature and high baryonic density, quarks form Cooper pairs due to the existence of attractive interactions among them. The diquark condensate modifies the ground state of QCD and leads to the phenomenon of color superconductivity [1-4]. This phase is typically characterized by the Anderson-Higgs mechanism and an energy gap associated with the fermionic quasiparticles. It is expected that this state of matter is realized in compact stars. Studying the properties of cold dense quark matter may explain some basic features of neutron stars and could even lead to the prediction of new stellar objects (see [4] for a recent review on the subject).

A lot of progress has been made in the microscopic understanding of color superconductivity. Important open questions concern macroscopic properties of dense quark matter. Transport theory is known to provide a very efficient framework for the study of low-energy and long-range fluctuations in a medium. In this Letter, we present a transport equation for the gapped quasiparticle excitations of the two-flavor color superconductor. This is a convenient starting point for both the construction of low-energy effective theories and the computation of macroscopic observables within the kinetic or hydrodynamical limits.

The global properties of a color superconductor depend dramatically on the number of quark flavors $N_{f}$ participating in the condensation. Here we shall discuss the case of two flavors $N_{f}=2$, which exhibits a very rich structure. The diquark condensate breaks the color SU(3) group into an $\mathrm{SU}(2)$ subgroup. As a result, five gluons acquire masses through the Anderson-Higgs mechanism, while three of them remain massless. Also not all the quarks attain a gap. In the high-density limit, and because of asymptotic freedom, the gap and the gluon masses can be computed reliably from first principles [5-11]. A well-defined hierarchy of scales then appears: at weak coupling $g \ll 1$, the gap $\Delta$ is exponentially smaller than the gluon masses $\sim g \mu$ and the chemical potential $\mu$.
PACS numbers: $12.38 . \mathrm{Mh}, 24.85 .+\mathrm{p}$

The low-energy physics of a two-flavor color superconductor is dominated by its light degrees of freedom. At vanishing temperature, these are the massless gauge bosons, the gapless quarks, and a (pseudo-) Goldstone boson, similar to the $\eta$ meson. However, the gapless quarks and the $\eta$ meson are neutral with respect to the unbroken SU(2) subgroup. In turn, the condensate, although neutral with respect to the unbroken $\mathrm{SU}(2)$, polarizes the medium since their constituents carry SU(2) charges. Hence, the dynamics of the light $\mathrm{SU}(2)$ gauge fields differs from the vacuum theory. This picture has recently been introduced by Rischke, Son, and Stephanov [12]. Their infrared effective theory for momenta $k \ll \Delta$ is

$$
S_{\text {eff }}^{T=0}=\int d^{4} x\left(\frac{\epsilon}{2} \mathbf{E}_{a} \cdot \mathbf{E}_{a}-\frac{1}{2 \lambda} \mathbf{B}_{a} \cdot \mathbf{B}_{a}\right),
$$

where $E_{i}^{a} \equiv F_{0 i}^{a}$ and $B_{i}^{a} \equiv \frac{1}{2} \epsilon_{i j k} F_{j k}^{a}$ are the $\mathrm{SU}(2)$ electric and magnetic fields. The constants $\epsilon$ and $\lambda$ are the dielectric susceptibility and magnetic permeability of the medium. To leading order, $\lambda=1$ and $\epsilon=1+g^{2} \mu^{2} /\left(18 \pi^{2} \Delta^{2}\right)$ [12]. As a consequence, the velocity of the $\mathrm{SU}(2)$ gluons is smaller than in vacuum. This theory is confining, but the scale of confinement is highly reduced with respect to the one in vacuum with $\Lambda_{\mathrm{QCD}}^{\prime} \sim \Delta \exp \left(-\frac{2 \sqrt{2} \pi}{11} \frac{\mu}{g} \Delta\right)$ [12]. Because of asymptotic freedom, it is expected that perturbative computations are reliable for energy scales larger than $\Lambda_{\mathrm{QCD}}^{\prime}$.

At nonvanishing temperature, thermal excitations modify the low-energy physics. The condensate melts at the critical temperature $T_{c} \approx 0.567 \Delta_{0}$ [7] ( $\Delta_{0}$ is the gap at vanishing temperature). We restrict the discussion to temperatures within $\Lambda_{\mathrm{QCD}}^{\prime} \ll T<T_{c}$, which provides the basis for the perturbative computations below. In this regime, the main contribution to the long distance properties of the SU(2) fields stems from the thermal excitations of the constituents of the diquark condensate. The thermal excitations of the massless gauge fields contribute only at the order $g^{2} T^{2}$ and are subleading for sufficiently large $\mu$. Those of the gapless quarks and of the $\eta$ meson do not couple to the SU(2) gauge fields. 
The thermal excitations due to the constituents of the diquark condensate display a quasiparticle structure. This implies that they can be cast into a transport equation. To that end, and working in natural units $k_{B}=\hbar=c=1$, we introduce the on-shell one-particle phase space density $f(x, \mathbf{p}, Q), x^{\mu}=(t, \mathbf{x})$, describing the quasiparticles. The distribution function depends on time, the phase space variables position $\mathbf{x}$, momentum $\mathbf{p}$, and on $\mathrm{SU}(2)$ color charges $Q_{a}$, with the color index $a=1,2$, and 3 . The quasiparticles carry $\mathrm{SU}(2)$ color charges simply because the constituents of the condensate do. The on-shell condition for massless quarks $m_{q}=0$ relates the energy of the quasiparticle excitation to the chemical potential and the gap as $p_{0} \equiv \epsilon_{p}=\sqrt{(p-\mu)^{2}+\Delta^{2}(T)}$. The gap is both temperature and momentum dependent. From now on, we can neglect its momentum dependence which is a subleading effect. The velocity of the quasiparticles is given by

$$
\mathbf{v}_{p} \equiv \frac{\partial \boldsymbol{\epsilon}_{p}}{\partial \mathbf{p}}=\frac{|p-\mu|}{\sqrt{(p-\mu)^{2}+\Delta^{2}(T)}} \hat{\mathbf{p}}
$$

and depends on both the chemical potential and the gap. For $\Delta=0$, the quasiparticles would travel at the speed of light. However, in the presence of the gap $\Delta \neq 0$, their propagation is suppressed, $v_{p} \equiv\left|\mathbf{v}_{p}\right| \leq 1$.

The one-particle distribution function $f(x, \mathbf{p}, Q)$ obeys a very simple transport equation, given by

$$
\left[D_{t}+\mathbf{v}_{p} \cdot \mathbf{D}-g Q_{a}\left(\mathbf{E}^{a}+\mathbf{v}_{p} \times \mathbf{B}^{a}\right) \frac{\partial}{\partial \mathbf{p}}\right] f=C[f] .
$$

Here we have introduced the shorthand notation $D_{\mu} f \equiv$ $\left[\partial_{\mu}-g \epsilon^{a b c} Q_{c} A_{b}^{\mu} \partial / \partial Q^{a}\right] f$ for the covariant derivative acting on $f$. The first two terms on the left-hand side of Eq. (3) combine to a covariant drift term $v_{p}^{\mu} D_{\mu}$, where $v_{p}^{\mu}=\left(1, \mathbf{v}_{p}\right)$ and $D_{\mu}=\left(D_{t}, \mathbf{D}\right)$. The terms proportional to the color electric and magnetic fields provide a force term. The right-hand side of Eq. (3) contains a (yet unspecified) collision term $C[f]$.

The thermal quasiparticles carry an $\mathrm{SU}(2)$ charge, and hence provide an $\mathrm{SU}(2)$ color current. It is given by

$$
J_{a}^{\mu}(x)=g \sum_{\substack{\text { helicities } \\ \text { species }}} \int \frac{d^{3} p}{(2 \pi)^{3}} d Q v_{p}^{\mu} Q_{a} f(x, \mathbf{p}, Q) .
$$

Below, we simply omit a species or helicity index on $f$, as well as the explicit sum over them. The color measure is normalized $\int d Q=1$, and obeys $\int d Q Q_{a}=0$ and $\int d Q Q_{a} Q_{b}=C_{2} \delta_{a b}$, where $C_{2}$ denotes the quadratic Casimir ( $C_{2}=\frac{1}{2}$ for quarks in the fundamental). The color current Eq. (4) is covariantly conserved for $C[f]=0$. For $C[f] \neq 0$ a covariantly conserved current implies certain restrictions in the form of the collision term.

Transport equations similar to Eq. (3) have been known previously: (i) The analog of Eq. (3) at high temperature for $\mu=\Delta=0$ [and $\mathrm{SU}(N)$ color charges $Q_{a}$ ] has been introduced in [13] for the description of the quark-gluon plasma at high temperature. In the Vlasov approximation and for $C[f]=0$, it reproduces the hard thermal loop (HTL) effective theory [14] to leading order in $g$ [15]. (ii) A formalism which allows one to go systematically beyond the Vlasov approximation has been derived as well [16]. This includes the derivation of the collision term $C[f]$. (iii) At high density and $T=\Delta=0$, the corresponding transport equation leads to the hard dense loop (HDL) effective theory for $C[f]=0$, and to leading order in $g$ [17]. (iv) In the nonrelativistic limit for the Abelian case, our transport equation reduces to the one for a BCS superconductor [18].

In the remaining part of the Letter, we study the collisionless dynamics $C[f]=0$ of the color superconductor close to thermal equilibrium and to leading order in the gauge coupling. Consider the distribution function $f(x, \mathbf{p}, Q)=f^{\mathrm{eq}}\left(p_{0}\right)+g f^{(1)}(x, \mathbf{p}, Q)$. Here $f^{\mathrm{eq}}\left(p_{0}\right)=$ $1 /\left[\exp \left(\epsilon_{p} / T\right)+1\right]$ is the fermionic equilibrium distribution function and $g f^{(1)}(x, \mathbf{p}, Q)$ describes a slight deviation from equilibrium. For convenience, we also introduce the color density

$$
J_{a}(x, \mathbf{p})=g \int d Q Q_{a} f(x, \mathbf{p}, Q),
$$

from which the induced color current of the medium Eq. (4) follows as $J_{a}^{\rho}(x)=\int \frac{d^{3} p}{(2 \pi)^{3}} v_{p}^{\rho} J_{a}(x, \mathbf{p})$. Expanding the transport equation (3) to leading order in $g$, and taking the two helicities per quasiparticle into account, we find the transport equation for the color density as

$$
\left[D_{t}+\mathbf{v}_{p} \cdot \mathbf{D}\right] J(x, \mathbf{p})=g^{2} N_{f} \mathbf{v}_{p} \cdot \mathbf{E}(x) \frac{d f^{\mathrm{eq}}}{d \epsilon_{p}} .
$$

The solution of the transport equation reads

$$
J_{a}^{\mu}(x)=g^{2} N_{f} \int \frac{d^{3} p d^{4} y}{(2 \pi)^{3}} v_{p}^{\mu} G_{a b} \mathbf{v}_{p} \cdot \mathbf{E}_{b}(y) \frac{d f^{\mathrm{eq}}}{d \epsilon_{p}},
$$

with the Greens function $G_{a b} \equiv\left\langle y\left|1 /\left(v_{p} \cdot D\right)\right| x\right\rangle_{a b}$. After having solved the transport equation, the relevant information concerning the low-energy effective theory is contained in the functional $J[A]$. Notice that the above derivation is analogous to the derivation of the HTL and HDL effective theories from kinetic theory [14,15,17]. Owing to this resemblance, we call the diagrams, which are derived from Eq. (7), hard superconducting loops (HSL). The HSL effective action follows from Eq. (7), by solving $J[A]=-\delta \Gamma_{\mathrm{HSL}}[A] / \delta A$ for $\Gamma_{\mathrm{HSL}}[A]$, and all HSL diagrams can be derived by performing functional derivatives to the effective action (or the induced current). We thus reach the conclusion that the low-energy effective theory for a two-flavor color superconductor at finite temperature reads $S_{\text {eff }}^{T}=S_{\text {eff }}^{T=0}+\Gamma_{\mathrm{HSL}}$ to leading order in $g$. This theory is effective for modes with $k \ll \Delta$.

Let us have a closer look into the induced current, which we formally expand as $J_{\mu}^{a}[A]=\prod_{\mu \nu}^{a b} A_{b}^{\nu}+$ $\frac{1}{2} \Gamma_{\mu \nu \rho}^{a b c} A_{b}^{\nu} A_{c}^{\rho}+\ldots$ in powers of the gauge fields. The most relevant information on the thermal effects is contained in the thermal polarization tensor $\Pi_{\mu \nu}^{a b}$. Using Eq. (7), we find 


$$
\begin{aligned}
\Pi_{a b}^{\mu \nu}(k)= & g^{2} N_{f} \delta_{a b} \\
& \times \int \frac{d^{3} p}{(2 \pi)^{3}} \frac{d f^{\mathrm{eq}}}{d \epsilon_{p}}\left(g^{\mu 0} g^{\nu 0}-k_{0} \frac{v_{p}^{\mu} v_{p}^{\nu}}{k \cdot v_{p}}\right) .
\end{aligned}
$$

It obeys the Ward identity $k_{\mu} \Pi_{a b}^{\mu \nu}(k)=0$. With retarded boundary conditions $k_{0} \rightarrow k_{0}+i 0^{+}$, the polarization tensor has an imaginary part,

$$
\begin{aligned}
\operatorname{Im}_{a b}^{\mu \nu}(k)= & \delta_{a b} \pi g^{2} N_{f} k_{0} \\
& \times \int \frac{d^{3} p}{(2 \pi)^{3}} \frac{d f^{\mathrm{eq}}}{d \epsilon_{p}} v_{p}^{\mu} v_{p}^{\nu} \delta\left(k \cdot v_{p}\right),
\end{aligned}
$$

which corresponds to Landau damping. Performing the angular integration, we obtain for the longitudinal and transverse projections of the polarization tensor

$$
\begin{aligned}
\Pi_{L}(k)= & \frac{g^{2} N_{f}}{2 \pi^{2}} \int_{0}^{\infty} d p p^{2} \frac{d f^{\mathrm{eq}}}{d \epsilon_{p}}\left[1-\frac{1}{2} \frac{k_{0}}{k v_{p}}\right. \\
& \left.\times\left(\ln \left|\frac{k_{0}+k v_{p}}{k_{0}-k v_{p}}\right|-i \pi \Theta\left(k^{2} v_{p}^{2}-k_{0}^{2}\right)\right)\right],
\end{aligned}
$$

$$
\begin{aligned}
\Pi_{T}(k)= & \frac{g^{2} N_{f}}{4 \pi^{2}} \frac{k_{0}^{2}}{k^{2}} \int_{0}^{\infty} d p p^{2} \frac{d f^{\mathrm{eq}}}{d \epsilon_{p}}\left[1+\frac{1}{2}\left(\frac{k v_{p}}{k_{0}}-\frac{k_{0}}{k v_{p}}\right)\right. \\
& \left.\times\left(\ln \left|\frac{k_{0}+k v_{p}}{k_{0}-k v_{p}}\right|-i \pi \Theta\left(k^{2} v_{p}^{2}-k_{0}^{2}\right)\right)\right],
\end{aligned}
$$

where $\Theta$ is the step function. We first consider the real part of the polarization tensor. From Eq. (8), and in the limit $k_{0} \rightarrow 0$, we infer that the longitudinal gauge bosons acquire a thermal mass, the Debye mass, while the transverse ones remain massless. The (square of the) Debye mass is given by

$$
m_{D}^{2}=-\frac{g^{2} N_{f}}{2 \pi^{2}} \int_{0}^{\infty} d p p^{2} \frac{d f^{\mathrm{eq}}}{d \epsilon_{p}} \equiv M^{2} I_{0}\left(\frac{\Delta}{T}, \frac{T}{\mu}\right) .
$$

For convenience, we have factored out the Debye mass $M$ of the ultradegenerate plasma in the normal phase, $M^{2} \equiv$ $g^{2} N_{f} \mu^{2} / 2 \pi^{2}$. The dimensionless functions

$$
I_{n}\left(\frac{\Delta}{T}, \frac{T}{\mu}\right)=-\frac{1}{\mu^{2}} \int_{0}^{\infty} d p p^{2} \frac{d f^{\mathrm{eq}}}{d \epsilon_{p}} v_{p}^{n}
$$

obey $I_{n} \geq I_{n+1}>0$ for all $n$ due to $v_{p} \leq 1$. Equality holds for vanishing gap. For the physically relevant range of parameters $T<\Delta \ll \mu$, the functions $I_{n}$ are $\ll 1$. In particular, it is easy to see that $I_{n}(\infty, 0)=0$ : there is no Debye screening for the $\mathrm{SU}(2)$ gluons at $T=0$ in the superconducting phase. In the limit where $\Delta / T \gg 1$, and to leading order in $T / \mu \ll 1$, the Debye mass reduces to

$$
m_{D}^{2}=M^{2} \sqrt{2 \pi \frac{\Delta}{T}} \exp (-\Delta / T)
$$

The dispersion relations for the longitudinal and transverse gluons follow from the poles of the corresponding propagators,

$$
\begin{array}{r}
\epsilon k^{2}-\left.\operatorname{Re}_{L}\left(k_{0}, k\right)\right|_{k_{0}=\omega_{L}(k)}=0, \\
\epsilon k_{0}^{2}-\frac{1}{\lambda} k^{2}+\left.\operatorname{Re}_{T}\left(k_{0}, k\right)\right|_{k_{0}=\omega_{T}(k)}=0,
\end{array}
$$

where $\epsilon$ and $\lambda$ have been introduced in Eq. (1). The plasma frequency $\omega_{\mathrm{pl}}$ follows from Eq. (12) as

$$
\omega_{\mathrm{pl}}^{2}=\frac{1}{3 \epsilon} M^{2} I_{2}\left(\frac{\Delta}{T}, \frac{T}{\mu}\right) .
$$

For generic external momenta the dispersion relations can be solved only numerically. In turn, if the spatial momenta are much smaller than the plasma frequency $k \ll \omega_{\text {pl }}$, solutions to Eq. (12) can be expanded in powers of $k^{2} / \omega_{\mathrm{pl}}^{2}$ as

$$
\begin{aligned}
& \omega_{L}^{2}(k)=\omega_{\mathrm{pl}}^{2}\left[1+\frac{3}{5} \frac{I_{4}}{I_{2}} \frac{k^{2}}{\omega_{\mathrm{pl}}^{2}}+\mathcal{O}\left(\frac{k^{4}}{\omega_{\mathrm{pl}}^{4}}\right)\right], \\
& \omega_{T}^{2}(k)=\omega_{\mathrm{pl}}^{2}\left[1+\left(\frac{1}{\epsilon \lambda}+\frac{1}{5} \frac{I_{4}}{I_{2}}\right) \frac{k^{2}}{\omega_{\mathrm{pl}}^{2}}+\mathcal{O}\left(\frac{k^{4}}{\omega_{\mathrm{pl}}^{4}}\right)\right] .
\end{aligned}
$$

Let us now consider the imaginary part of Eq. (8), which describes Landau damping. Since $v_{p} \leq 1$, we conclude that Landau damping occurs only for $k_{0}^{2} \leq k^{2}$. Hence, plasmon and transverse gluon excitations are stable as long as $\omega_{L, T}(k)>k$. Furthermore, we notice that the imaginary part of Eq. (8) is logarithmically divergent: the quasiparticle velocity vanishes for momenta close to the Fermi surface, which is an immediate consequence of the presence of a gap; cf. Eq. (2). This divergence does not appear in the real part, because the logarithm acts as a regulator for the $1 / v_{p}$ factor. To leading order in $T / \mu$, and in the region of small frequencies $k_{0}^{2} \ll k^{2}$, we find at logarithmic accuracy, and for all values of $\Delta / T$,

$$
\begin{aligned}
\operatorname{Im}_{L}= & -2 \pi M^{2} \frac{k_{0}}{k} \frac{\Delta}{T} \frac{\ln \left(k / k_{0}\right)}{\left(e^{\Delta / T}+1\right)\left(e^{-\Delta / T}+1\right)}, \\
\operatorname{Im}_{T}= & \pi M^{2} \frac{k_{0}}{k}\left[\frac{1}{e^{\Delta / T}+1}\right. \\
& \left.-2 \frac{k_{0}^{2}}{k^{2}} \frac{\Delta}{T} \frac{\ln k / k_{0}}{\left(e^{\Delta / T}+1\right)\left(e^{-\Delta / T}+1\right)}\right] .
\end{aligned}
$$

For small frequencies, Landau damping is dominated by the logarithmic terms, which are proportional to the gap. Once the gap vanishes, subleading terms in $\frac{\Delta}{T}$, not displayed in Eq. (15), take over and reduce Im $\Pi$ to known expressions for the normal phase.

Finally, we explain how the polarization tensor, as obtained within the present transport theory, matches the computation of $\Pi^{\mu \nu}$ for external momenta $k_{0}, k \ll \Delta$ to one-loop order from quantum field theory. The one-loop gluon self-energy for a two-flavor color superconductor has been computed by Rischke, and the polarization tensor 
for the unbroken $\mathrm{SU}(2)$ subgroup is given in Eq. (99) of [11]. It contains contributions from particle-particle, particle-antiparticle, and antiparticle-antiparticle excitations. The particle-antiparticle contribution to $\Pi^{00}$ and $\Pi^{0 i}$ at low external momenta, and the antiparticleantiparticle excitations are subleading. The particleparticle contributions divide into two types. The first ones have poles for gluonic frequencies $k_{0}= \pm\left(\epsilon_{p}+\epsilon_{p-k}\right)$ and an imaginary part once $k_{0}$ exceeds the Cooper pair binding energy $2 \Delta$. These terms are related to the formation or breaking of a Cooper pair, and suppressed for low external gluon momenta. The second type of terms, only nonvanishing for $T \neq 0$, have poles at $k_{0}= \pm\left(\epsilon_{p}-\epsilon_{p-k}\right)$. For $k \ll \Delta$ we approximate it by $k_{0} \approx \pm \frac{\partial \epsilon_{p}}{\partial \mathbf{p}} \cdot \mathbf{k}$. The prefactor, a difference of thermal distribution functions, is approximated by $f^{\mathrm{eq}}\left(\epsilon_{p}\right)-$ $f^{\mathrm{eq}}\left(\epsilon_{p-k}\right) \approx \frac{\partial \epsilon_{p}}{\partial \mathbf{p}} \cdot \mathbf{k} \frac{d f^{\mathrm{eq}}}{d \epsilon_{p}}$. After simple algebraic manipulations we finally end up with the result given above. We conclude that this part of the one-loop polarization tensor describes the collisionless dynamics of thermal quasiparticles for a two-flavor color superconductor. The same type of approximations can be carried out for $\Pi^{i j}$ to one-loop order. There, apart from the HSL contributions, additional terms arise due to particle-particle and particle-antiparticle excitations; cf. Eq. (112) of [11]. We have not evaluated these terms explicitly. However, we expect them to be subleading or vanishing, as otherwise the Ward identity $k_{\mu} \Pi_{a b}^{\mu \nu}(k)=0$ is violated. For $T=0$, this has been confirmed in [11].

To summarize, we have provided a transport equation for the gapped quarks of two-flavor color superconductors. Its simple structure is based on the quasiparticle behavior of the thermal excitations of the condensate, in consistency with the underlying quantum field theory. We have constructed a low temperature infrared effective theory of the superconductor. To leading order, we found Landau damping, and Debye screening of the chromoelectric fields. Beyond leading order, chromomagnetic fields are damped because they scatter with the quasiparticles. The damping rate is related to the color conductivity. It should be possible to compute the rate from the transport equation (3), amended by the relevant collision term. The latter can be derived, for example, using the methods developed in [16].

We have not discussed the transport equations for gapless quarks or the $\eta$ meson, because they do not carry SU(2) charges. However, their excitations are light compared to the gapped quasiparticles, and dominant for other transport properties such as thermal and electrical conductivities or shear viscosity. The corresponding set of transport equations will be discussed elsewhere. It would be very interesting to study the transport equations in a three-flavor color superconductor [19]. For $N_{f}=3$ the quark-quark condensate breaks the $\mathrm{SU}(3)$ gauge group completely, as well as some global flavor symmetries. Transport phenomena should then be dominated by the Goldstone modes associated with the breaking of the global symmetries. The corresponding transport equations will be substantially different for the two- and threeflavor cases.

This work has been supported by the European Community through the Marie-Curie Grants No. HPMF-CT-199900404 and No. HPMF-CT-1999-00391.

[1] B. C. Barrois, Nucl. Phys. B129, 390 (1977).

[2] D. Bailin and A. Love, Phys. Rep. 107, 325 (1984).

[3] M. Alford, K. Rajagopal, and F. Wilczek, Phys. Lett. B 422, 247 (1998); R. Rapp, T. Schäfer, E. V. Shuryak, and M. Velkovsky, Phys. Rev. Lett. 81, 53 (1998).

[4] K. Rajagopal and F. Wilczek, hep-ph/0011333.

[5] D. T. Son, Phys. Rev. D 59, 094019 (1999).

[6] T. Schäfer and F. Wilczek, Phys. Rev. D 60, 114033 (1999).

[7] R. D. Pisarski and D. H. Rischke, Phys. Rev. D 61, 051501 (2000); 61, 074017 (2000).

[8] D. K. Hong, Phys. Lett. B 473, 118 (2000); D. K. Hong, V. A. Miransky, I. A. Shovkovy, and L. C. Wijewardhana, Phys. Rev. D 61, 056001 (2000).

[9] W.E. Brown, J. T. Liu, and H. Ren, Phys. Rev. D 61, 114012 (2000); 62, 054016 (2000).

[10] C. Manuel, Phys. Rev. D 62, 114008 (2000).

[11] D. H. Rischke, Phys. Rev. D 62, 034007 (2000).

[12] D. H. Rischke, D. T. Son, and M. A. Stephanov, hep-ph/ 0011379.

[13] U. Heinz, Phys. Rev. Lett. 51, 351 (1983).

[14] R. D. Pisarski, Phys. Rev. Lett. 63, 1129 (1989); E. Braaten and R. D. Pisarski, Nucl. Phys. B337, 569 (1990); J. P. Blaizot and E. Iancu, Phys. Rev. Lett. 70, 3376 (1993); Nucl. Phys., B417, 608 (1994).

[15] P. F. Kelly, Q. Liu, C. Lucchesi, and C. Manuel, Phys. Rev. Lett. 72, 3461 (1994); Phys. Rev. D 50, 4209 (1994).

[16] D. F. Litim and C. Manuel, Phys. Rev. Lett. 82, 4981 (1999); Nucl. Phys. B562, 237 (1999).

[17] C. Manuel, Phys. Rev. D 53, 5866 (1996).

[18] E. Lifshitz and L. Pitaevskii, Physical Kinetics (Pergamon Press, Oxford, 1981).

[19] M. Alford, K. Rajagopal, and F. Wilczek, Nucl. Phys. B537, 443 (1999). 